\title{
Porphyrin accumulation in humans with common dysfunctional variants of $A B C G 2$, a porphyrin transporter: potential association with acquired photosensitivity
}

\author{
Masayuki Sakiyama ${ }^{1,2} \cdot$ Hirotaka Matsuo $^{1}$ (1) $\cdot$ Yu Toyoda $^{1}$ (D) Yuiko Yonekura ${ }^{2} \cdot$ Takahiro Ishikawa $^{2} \cdot$ \\ Akiyoshi Nakayama $^{1}$ (D) Toshihide Higashino $^{1} \cdot$ Yusuke Kawamura $^{1}\left[\right.$ Norihiro Fujimoto $^{2} \cdot$ Nariyoshi Shinomiya $^{1}$ (1) \\ Takahiro Satoh ${ }^{2}$ (1)
}

Received: 5 March 2021 / Accepted: 12 April 2021 / Published online: 19 May 2021

(c) The Author(s) 2021

\begin{abstract}
Photosensitivity is a skin reaction disorder mediated by phototoxic and/or photoallergic mechanisms. The accumulation of porphyrins is generally considered to induce phototoxicity. ATP-binding cassette subfamily G member 2 (ABCG2) has been identified as a transporter of porphyrins and its common variants-p.Gln126Ter (rs72552713) and p.Gln141Lys (rs2231142) - reportedly decrease the function of porphyrin transport in vitro; however, the physiological importance of ABCG2 as a porphyrin transporter remains to be fully elucidated. We herein investigated whether ABCG2 dysfunction could lead to porphyrin accumulation and photosensitivity in Japanese subjects, and found it to be significantly correlated with erythrocyte protoporphyrin levels $(P=0.012)$. This appears to be the first clinical finding of ABCG2 dysfunction-associated protoporphyrin accumulation in humans. We divided the patients into a chronic actinic dermatosis (CAD) group and a nonCAD group. CAD was diagnosed based on the criteria of reduced minimal erythema doses to ultraviolet B (UVB) and/or ultraviolet A (UVA). The non-CAD group was composed of patients who exhibited normal reactions to UVB and UVA on phototesting, but had histories of recurrent erythema/papules on sun-exposed areas. Estimated ABCG2 function according to $A B C G 2$ genotypes in the non-CAD group was significantly lower than in the general Japanese population $(P=0.045)$. In contrast, no difference was found in ABCG2 function between the CAD group and the general population, suggesting that ABCG2 dysfunction might be a genetic factor in non-CAD patients with clinical photosensitivity. In this context, genetic dysfunction of ABCG2 might be an overlooked pathological etiology of "photosensitivity of unknown cause."
\end{abstract}

Keywords $\mathrm{ABC}$ transporter $\cdot$ Genetic variations $\cdot$ Photodermatosis $\cdot$ Phototoxicity $\cdot$ Porphyrin transport

\section{Introduction}

Photosensitivity is a disorder of the skin caused by reactions that are mediated by phototoxic and/or photoallergic mechanisms in sun-exposed areas of the body. Phototoxic reactions of this nature, which derive from both environmental and genetic factors, are generally caused by drugs, metabolic disturbances, or genetic disorders [1,2]. Chronic actinic dermatosis (CAD) is a chronic eczematous skin disease of sun-exposed areas that are sensitive to ultraviolet B (UVB) and/or ultraviolet A (UVA) [3]; however, the molecular mechanisms that cause the disease remain unclear. In some cases, such as polymorphous light eruption (PLE), abnormal erythematous reaction such as from a reduced minimal erythema doses (MED) to UVA and UVB on phototests is often missed, despite the presence of clinical photosensitivity. 
Given the unknown etiology of such skin disorders, there is a growing need to investigate their underlying molecular mechanisms.

The accumulation of porphyrins is understood to be a trigger for phototoxicity, as seen in the examples of erythropoietic protoporphyria and porphyria cutanea tarda $[4,5]$. Membrane transport of porphyrins, an important process in their cellular handling, is reportedly mediated by porphyrin transporters [6]. One of these, a urate transporter ATP-binding cassette subfamily G member 2 (ABCG2, also known as breast cancer resistance protein, which has been wellcharacterized as a drug transporter [7]), has been identified as transporting some porphyrins $[8,9]$. In vitro functional assays have shown that two common variants of ABCG2p.Gln126Ter (p.Q126X: rs72552713) and p.Gln141Lys (p.Q141K: rs2231142) [10]—decrease the function of porphyrin transport [11]. Abcg2 knockout mice fed on a diet high in alfalfa exhibit severe phototoxicity on light-exposed skin; this diet-dependent phototoxicity is associated with the accumulation of pheophorbide a ( $\mathrm{Pa}$, a dietary chlorophyll breakdown product) [12]. $\mathrm{Pa}$ is a type of porphyrin and is an ABCG2 substrate, such as some porphyrins, including protoporphyrin IX, a major porphyrin in the blood. Although previous studies such as the above suggest the involvement of ABCG2 dysfunction in several porphyrin-related phototoxicities in mammals, there is little information available on the effects of ABCG2 dysfunction on porphyrin accumulation and photosensitivity in humans. To gain more insight into this issue, we herein investigated the relationship, in Japanese patients, between $A B C G 2$ genotypes and photosensitivity that lacks apparent causes.

\section{Methods}

\section{Study participants}

This study was approved by the National Defense Medical College's institutional ethics committee, and all procedures were performed in accordance with the Declaration of Helsinki. Written informed consent was obtained from each subject participating in this study before DNA samples and erythrocyte protoporphyrin data were collected.

The study participants comprised 23 Japanese patients (13 males and 10 females) with suspected photosensitivity based on their clinical histories and/or symptoms. Mean age (years) and standard deviation were 55.5 \pm 20.1 . Patients with systemic, metabolic, or genetic disorders, including porphyria, xeroderma pigmentosum, and systemic lupus erythematosus, were excluded. Those with drug-induced photosensitivity and photocontact dermatitis were also excluded. We performed phototests with UVB (280-380 nm) and UVA (300-430 nm) lamps of Dermaray-200 (Toshiba Medical
Supply, Tokyo, Japan). Seven patients were diagnosed with CAD based on the criteria of reduced MED to UVB $(50 \mathrm{~mJ} /$ $\left.\mathrm{cm}^{2}\right)$ and/or UVA $\left(10 \mathrm{~J} / \mathrm{cm}^{2}\right)$. Five CAD patients showed photosensitivity to both UVB and UVA; one CAD patient showed photosensitivity to UVA alone, and another CAD patient showed photosensitivity to UVB alone. The remaining 16 patients exhibited normal reactions to UVB and UVA on phototesting, but had histories of recurrent erythema/papules on sun-exposed areas; therefore, they were grouped as non-CAD patients.

\section{Genetic analysis}

Genomic DNA was extracted from whole peripheral blood cells. Genotyping of two $A B C G 2$ dysfunctional variants, p.Q126X (c.376C > T) and p.Q141K (c.421C > A), was performed using the TaqMan method (Thermo Fisher Scientific, Waltham, MA, USA) with a LightCycler 480 (Roche Diagnostics, Mannheim, Germany), as previously described [13]. Custom TaqMan assay probes were designed as follows. For p.Q126X, VIC-CCACTAATACTTACTTGT ACCAC and FAM-CCACTAATACTTACTTATACCAC; for p.Q141K, VIC-CTGCTGAGAACTGTAAGTT and FAMCTGCTGAGAACTTTAAGTT. To confirm their genotypes, DNA sequencing analysis was performed with the following primers. For p.Q126X, forward 5'-TGTACAATGAAAAGA GAAAGGTGAG-3' and reverse 5'-CTGCCTTTTCACATA AGTGTC-3'; for p.Q141K, forward 5'-ATGGAGTTAACT GTCAT TTGC-3' and reverse 5'-CACGTTCATATTATG TAACAAGCC-3'. Direct sequencing was performed with a 3130xl Genetic Analyzer (Thermo Fisher Scientific).

\section{Statistical analyses}

R software (version 3.1.1: http://www.r-project.org/) was used for all the statistical analysis calculations. Linear regression analysis and the Cochran-Armitage test were performed for the association analyses. The $P$ value of the Hardy-Weinberg equilibrium was calculated using the Chisquare test with Yates' correction. We set the significance threshold to $\alpha=0.05$.

\section{Results}

The call rates for two variants of $A B C G 2$ (p.Q126X and p.Q141K) were $100 \%$; these variants were in Hardy-Weinberg equilibrium $(P>0.05)$. Reportedly, p.Q126X is a nonfunctional variant and p.Q141K, which halves the protein levels of ABCG2, is a semi-functional variant for protoporphyrin transport [11]. Since there was no simultaneous presence of the minor alleles of p.Q126X and p.Q141K in one haplotype [10], three haplotypes: *1 (Q126 and Q141, the 
wild-type with full function), *2 (Q126 and K141), and *3 (X126 and Q141) were defined in this study as previously reported [14]. According to the degree of ABCG2 function based on these haplotypes, all the patients could be divided into the following groups: full function $(* 1 / * 1), 3 / 4$ function (mild dysfunction; $* 1 / * 2$ ), and $\leq 1 / 2$ function (moderate-tosevere dysfunction; $* 1 / * 3, * 2 / * 2, * 2 / * 3$, or $* 3 / * 3)[14,15]$, as shown in Table 1.

In the 23 patients with photosensitivity that lacked apparent causes, lower ABCG2 function was significantly correlated with higher erythrocyte protoporphyrin levels $(P=0.012$; Fig. 1), suggesting that ABCG2 dysfunction can lead to the accumulation of protoporphyrin in humans. We next assessed the association of ABCG2 dysfunction with photosensitivity. For this purpose, we divided the patients into CAD and non-CAD groups, as shown in Table 1. When the non-CAD group was compared with the general Japanese population studied in our previous report [13], the proportions of ABCG2 dysfunctional subjects were significantly different $(P=0.045$; Table 1). Whereas there were a greater number of ABCG2-dysfunctional subjects in the non-CAD group, this difference was not observed between the CAD patients and the general Japanese population $(P=0.82)$.

\section{Discussion}

In this study, we showed, for the first time to the best of our knowledge, a positive association between genetic dysfunction of ABCG2 and increased erythrocyte protoporphyrin levels in humans, which might result in the potentially higher risk of non-CAD photosensitivity in ABCG2-deficient subjects. Given the intestinal role of $A B C G 2$ as a gatekeeper of ABCG2 substrates [16], together with the fact that ABCG2 also mediates the efflux of protoporphyrin IX from red blood

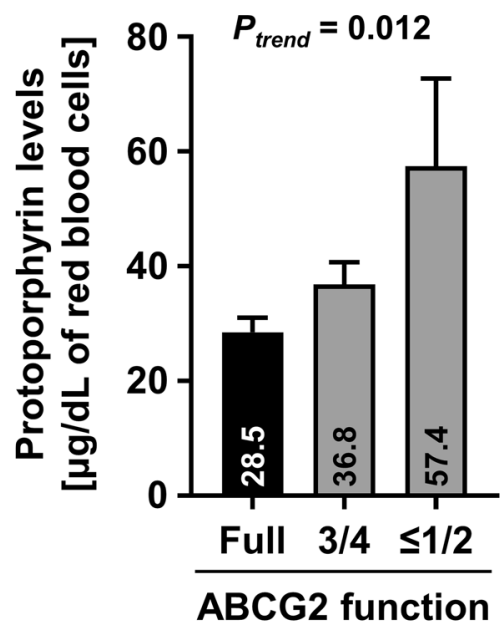

Fig. 1 Association between genetic dysfunction of $A B C G 2$ and higher erythrocyte protoporphyrin levels in photosensitive patients. The degrees of ABCG2 function were determined for 23 patients with photosensitivity based on their genotypes of two common dysfunctional variants of $A B C G 2, \mathrm{p} . \mathrm{Q} 126 \mathrm{X}$ and p.Q141K. Data are expressed as mean (which is given within the bars) \pm SEM. The $P$ value was calculated using linear regression analysis

cells into the blood, our findings provide clinical evidence that supports the commonly accepted notion that ABCG2 would regulate porphyrin handling in humans.

This study will also improve our understanding of the genetics-associated risk of diet-induced photosensitivity. Porphyrins are present in natural health supplements, including dried algae. Taking these supplements in large quantities may, therefore, increase the accumulation of porphyrins, which can theoretically raise the risk of photosensitivity in certain individuals, especially those with dysfunctional alleles of $A B C G 2$. Ingestion of chlorella tablets has induced phototoxic dermatitis in some
Table 1 Estimated ABCG2 function based on $A B C G 2$ genotypes in photosensitive patients and a general Japanese population

\begin{tabular}{lllll}
\hline $\begin{array}{l}\text { Estimated ABCG2 } \\
\text { function }\end{array}$ & Diplotype $^{\mathrm{a}}$ & CAD patients & $\begin{array}{l}\text { Non-CAD photosensi- } \\
\text { tive patients }\end{array}$ & $\begin{array}{l}\text { General } \\
\text { Japanese } \\
\text { population }\end{array}$ \\
\hline Full function & $* 1 / * 1$ & $4(57.1 \%)$ & $4(25.0 \%)$ & $253(50.6 \%)$ \\
$3 / 4$ function & $* 1 / * 2$ & $2(28.6 \%)$ & $8(50.0 \%)$ & $177(35.4 \%)$ \\
$\leq 1 / 2$ function & $* 2 / * 2, * 3 / * 1, * 3 / * 2$ & $1(14.3 \%)$ & $4(25.0 \%)$ & $70(14.0 \%)$ \\
Total & or *3/*3 & $7(100 \%)$ & $16(100 \%)$ & $500(100 \%)$ \\
& - & $P=0.82^{\mathrm{c}}$ & $P=0.045^{\mathrm{c}}$ & Reference $[13]$ \\
\hline
\end{tabular}

$C A D$ chronic actinic dermatosis

a*1, *2 and *3 represent haplotypes "C-C" (Q126 and Q141), "C-A" (Q126 and K141) and "T-C" (X126 and Q141) of two dysfunctional variants, p.Q126X (c.376C > T; rs72552713) and p.Q141K (c.421C>A; rs2231142), respectively

${ }^{b}$ Data on the general Japanese population were obtained from our previous report [13]

${ }^{c} P$ values were calculated using a trend test (Cochran-Armitage test) compared with the general Japanese population 
individuals with unknown $A B C G 2$ genotypes: $\mathrm{Pa}$ and its ester were identified as photosensitizing agents present in the tablets [17]. We also speculate that an ordinary diet can influence the clinical symptoms of photosensitivity in individuals harboring dysfunctional $A B C G 2$ alleles. This possibility may, at least partially, explain the negative (non-disease) results of phototests observed in patients in the non-CAD group, despite their histories of the clinical photosensitivity.

Before closing, some limitations warrant mentioning. First, because the non-CAD patient group likely consisted of a heterogeneous population of patients, further studies on more patients with photosensitivity will be needed. Second, we were not able to perform neither phototests with visible light nor photoprovocation tests using repeated exposures or larger single doses of UVB/UVA to address whether the patients could be diagnosed with PLE. Nevertheless, our data suggest that ABCG2 dysfunction may be an overlooked etiology of "photosensitivity of unknown cause" and that it might also affect the severity of phototoxic photosensitivity.

In summary, we report the increased erythrocyte protoporphyrin levels associated with ABCG2 dysfunction. Because genetic variations in $A B C G 2$ can affect its porphyrin transport activity, our study contribute to a better understanding of the genetic factors that influence individual differences in photosensitivity.

Acknowledgements The authors are deeply grateful to all the patients who participated in this study. We are also indebted to K. Gotanda, M. Miyazawa, S. Shimizu, K. Morichika, and M. Kawaguchi (National Defense Medical College) for genetic analysis as well as to A. Yoshii, R. Watanabe, Y. Imanaga, and S. Matsuo (National Defense Medical College) for sample collection.

Author contributions Conceptualization, MS and HM; validation, MS, YT, and AN; investigation, MS, HM, YT, YY, TI, AN, TH, YK, and NF (i.e., MS, YY, TI, and NF collected samples and analyzed the clinical data; MS, HM, AN, TH, and YK performed the genetic analyses; MS, HM, and YT performed the statistical analyses); data curation, MS; writing — original draft preparation, MS, HM, and YT; writing-review and editing, HM, YT, and TS; visualization, MS and YT; supervision, NS and TS; project administration, HM and TS; funding acquisition, HM and TS. All authors have read and agreed to the published version of the manuscript.

Funding This study was supported by JSPS KAKENHI Grant Numbers (25293145, 17H04128, and 20H00566), the Ministry of Defense of Japan, and the Kawano Masanori Memorial Foundation for Promotion of Pediatrics.

\section{Declarations}

Conflict of interest H.M. and N.S. have a patent pending based on the work reported in this paper. The other authors declare no conflicts of interest. The funders had no role in the design of the study; in the collection, analyses, or interpretation of data; in the writing of the manuscript, or in the decision to publish the results.
Ethical approval The study was conducted according to the guidelines of the Declaration of Helsinki, and approved by the Institutional Ethics Committee of the National Defense Medical College (No. 2364; approved on $2015 / 10 / 27$ )

Informed consent Informed consent was obtained from all subjects involved in the study.

Open Access This article is licensed under a Creative Commons Attribution 4.0 International License, which permits use, sharing, adaptation, distribution and reproduction in any medium or format, as long as you give appropriate credit to the original author(s) and the source, provide a link to the Creative Commons licence, and indicate if changes were made. The images or other third party material in this article are included in the article's Creative Commons licence, unless indicated otherwise in a credit line to the material. If material is not included in the article's Creative Commons licence and your intended use is not permitted by statutory regulation or exceeds the permitted use, you will need to obtain permission directly from the copyright holder. To view a copy of this licence, visit http://creativecommons.org/licenses/by/4.0/.

\section{References}

1. Monteiro AF, Rato M, Martins C. Drug-induced photosensitivity: photoallergic and phototoxic reactions. Clin Dermatol. 2016;34:571-81.

2. Millard TP, Hawk JL. Photosensitivity disorders: cause, effect and management. Am J Clin Dermatol. 2002;3:239-46.

3. Lim HW, Morison WL, Kamide R, Buchness MR, Harris R, Soter NA. Chronic actinic dermatitis. An analysis of 51 patients evaluated in the United States and Japan. Arch Dermatol. 1994;130:1284-9.

4. Dawe R. An overview of the cutaneous porphyrias. F1000Res. 2017;6:1906.

5. Norman RA. Past and future: porphyria and porphyrins. Skinmed. 2005;4:287-92.

6. Krishnamurthy P, Xie T, Schuetz JD. The role of transporters in cellular heme and porphyrin homeostasis. Pharmacol Ther. 2007; 114:345-58

7. Toyoda Y, Takada T, Suzuki H. Inhibitors of human ABCG2: from technical background to recent updates with clinical implications. Front Pharmacol. 2019;10:208.

8. Robey RW, Steadman K, Polgar O, Bates SE. ABCG2-mediated transport of photosensitizers: potential impact on photodynamic therapy. Cancer Biol Ther. 2005;4:187-94.

9. Zhou S, Zong Y, Ney PA, Nair G, Stewart CF, Sorrentino BP. Increased expression of the Abcg2 transporter during erythroid maturation plays a role in decreasing cellular protoporphyrin IX levels. Blood. 2005;105:2571-6.

10. Matsuo H, Takada T, Ichida K, et al. Common defects of ABCG2, a high-capacity urate exporter, cause gout: a function-based genetic analysis in a Japanese population. Sci Transl Med. 2009;1(5):5ra11.

11. Tamura A, Watanabe M, Saito $\mathrm{H}$, et al. Functional validation of the genetic polymorphisms of human ATP-binding cassette (ABC) transporter ABCG2: identification of alleles that are defective in porphyrin transport. Mol Pharmacol. 2006;70:287-96.

12. Jonker JW, Buitelaar M, Wagenaar E, et al. The breast cancer resistance protein protects against a major chlorophyll-derived dietary phototoxin and protoporphyria. Proc Natl Acad Sci USA. 2002;99:15649-54.

13. Sakiyama M, Matsuo H, Takada Y, et al. Ethnic differences in ATPbinding cassette transporter, sub-family G, member 2 (ABCG2/ BCRP): genotype combinations and estimated functions. Drug Metab Pharmacokinet. 2014;29:490-2. 
14. Matsuo H, Nakayama A, Sakiyama M, et al. ABCG2 dysfunction causes hyperuricemia due to both renal urate underexcretion and renal urate overload. Sci Rep. 2014;4:3755.

15. Ichida K, Matsuo H, Takada T, et al. Decreased extra-renal urate excretion is a common cause of hyperuricemia. Nat Commun. 2012;3:764.

16. Vlaming ML, Lagas JS, Schinkel AH. Physiological and pharmacological roles of ABCG2 (BCRP): recent findings in Abcg2 knockout mice. Adv Drug Deliv Rev. 2009;61:14-25.
17. Jitsukawa K, Suizu R, Hidano A. Chlorella photosensitization. New phytophotodermatosis. Int J Dermatol. 1984;23:263-8.

Publisher's Note Springer Nature remains neutral with regard to jurisdictional claims in published maps and institutional affiliations. 